\title{
Benefit of "Push-pull" Locomotion for Planetary Rover Mobility
}

\author{
C. Creager ${ }^{1}$, S. Moreland ${ }^{2}$, K. Skonieczny ${ }^{3}$, K. Johnson ${ }^{4}$, V. Asnani ${ }^{5}$, R. Gilligan ${ }^{6}$ \\ ${ }^{1}$ NASA Glenn Research Center, Mail Stop 23-3, 21000 Brookpark Road, Cleveland, \\ OH 44135; Ph (216) 433-6303; email: colin.m.creager@nasa.gov \\ ${ }^{2}$ Carnegie Mellon University, Robotics Institute, 5000 Forbes Ave., Pittsburgh, PA \\ 15213; Ph (412) 268-3856; email: smoreland@cmu.edu \\ ${ }^{3}$ Carnegie Mellon University, Robotics Institute; Ph (412) 268-9576; email: \\ kskoniec@andrew.cmu.edu \\ ${ }^{4}$ NASA Glenn Research Center, Mail Stop 23-3; Ph (216) 433-3571; email: \\ kyle.a.johnson@nasa.gov \\ ${ }^{5}$ NASA Glenn Research Center, Mail Stop 23-3; Ph (216) 433-3992; email: \\ vivake.m.asnani@nasa.gov \\ ${ }^{6}$ Ohio State University, 154 W. $12^{\text {th }}$ Ave., Columbus, Ohio 43210; Ph (614) 292- \\ 6446; email: gilligan.31@ buckeyemail.osu.edu
}

\section{ABSTRACT}

As NASA's exploration missions on planetary terrains become more aggressive, a focus on alternative modes of locomotion for rovers is necessary. In addition to climbing steep slopes, the terrain in these extreme environments is often unknown and can be extremely hard to traverse, increasing the likelihood of a vehicle or robot becoming damaged or immobilized. The conventional driving mode in which all wheels are either driven or free-rolling is very efficient on flat hard ground, but does not always provide enough traction to propel the vehicle through soft or steep terrain. This paper presents an alternative mode of travel and investigates the fundamental differences between these locomotion modes. The methods of "pushpull" locomotion discussed can be used with articulated wheeled vehicles and are identified as "walking" or "inching/inch-worming". In both cases, the braked nonrolling wheels provide increased thrust.

An in-depth study of how soil reacts under a rolling wheel vs. a braked wheel was performed by visually observing the motion of particles beneath the surface. This novel technique consists of driving or dragging a wheel in a soil bin against a transparent wall while high resolution, high-rate photographs are taken. Optical flow software was then used to determine shearing patterns in the soil. Different failure modes were observed for the rolling and braked wheel cases. A quantitative comparison of inching vs. conventional driving was also performed on a full-scale vehicle through a series of drawbar pull tests in the Lunar terrain strength simulant, GRC-1. The effect of tire stiffness was also compared; typically compliant tires provide better traction when driving in soft soil, however it's been observed that rigid wheels may provide better thrust when non-rolling. Initial tests indicate up to a possible $40 \%$ increase in pull force capability at high slip when inching vs. rolling. 


\section{INTRODUCTION}

The most conventional mode of locomotion for roving vehicles, referred to here as "rolling", consists of a set of wheels converting torque at the wheel centers to thrust at the ground contact point. This mode of driving has proven to be very efficient on flat hard ground; however, as NASA's missions to the Moon and Mars become more aggressive, driving conditions become much more difficult. Soft terrain, uneven surfaces, and steep slopes all present mobility challenges that must be achieved in order for a mission to succeed. To reduce the risk of becoming immobilized and allow for a greater range of terrains to be traversed, alternative modes of locomotion must be considered. The mode discussed here is referred to as "push-pull locomotion" and incorporates a different method of thrust generation.

\section{CONCEPT OF "PUSH-PULL LOCOMOTION"}

There are several variations to push-pull locomotion; however the principle is common to all. Unlike conventional rolling where thrust is produced by a rotating implement, the thrust force for push-pull locomotion is generated by keeping a portion of the vehicle stationary relative to the ground and re-positioning another segment to a different location by active articulation. In this way each vehicle segment is re-positioned resulting in a translation of the entire vehicle. During this cycle, the stationary implements in contact with the terrain are essentially "pushing" or "pulling" the vehicle while gripping the ground.

The most common form of push-pull locomotion is walking. An example of this is the ATHLETE Rover (NASA Jet Propulsion Laboratory), seen in Figure 1. The ATHLETE Rover consists of six legs. At the end of each leg is a wheel; each wheel can be lifted off of the ground and extended outward, then lowered giving it the capability to walk.

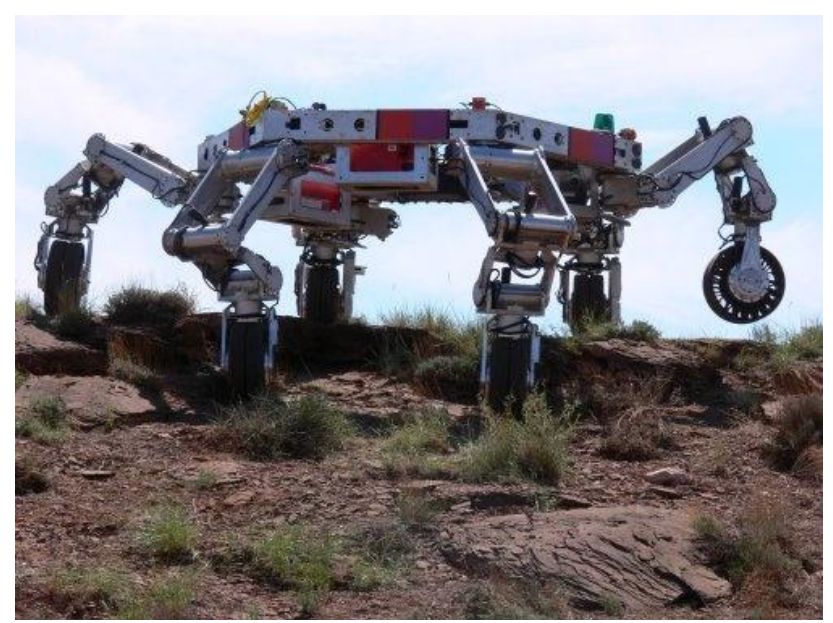

Figure 1: The ATHLETE Rover (NASA Jet Propulsion Laboratory) demonstrating "walking"

The variation of push-pull locomotion that is focused on in this paper is called "inching" (or "inch-worming"). It is visually similar to the method an inch-worm uses to propel itself forward and uses a combination of rolling wheels and vehicle 
articulation. The Scarab roving vehicle (Wettergreen, 2010), developed at Carnegie Mellon University, is a four wheel drive robotic vehicle with the ability to inch. On each side, each wheel is attached to the end of an arm that extends out from the center of the chassis at a shoulder joint. An actuator controls the angle between these arms, thus creating the ability to vary the wheel base (distance between the front and rear wheels). When inching, the rear wheels are held in place relative to the ground while the wheel base is increased and the front tires are driven forward (see Figure 2a). Once the front wheels are in place, the back wheels are driven forward while the wheel base is reduced (Figure 2b). During this cycle, two tires are always stationary relative to the ground acting as anchors from which the rest of the vehicle can push or pull itself in to position.

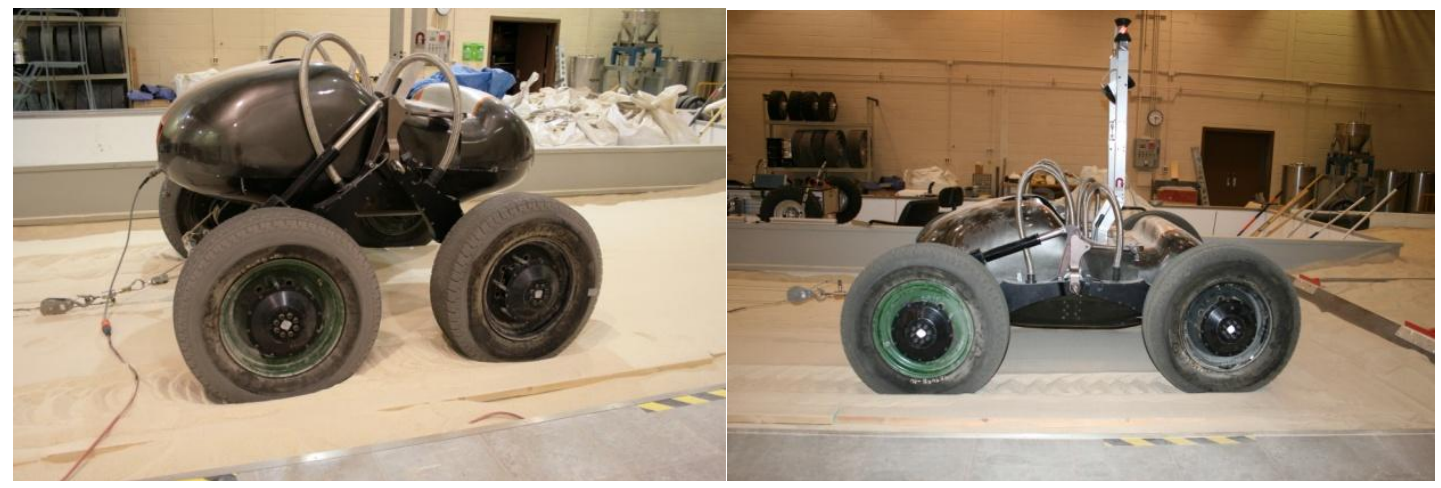

Figure 2: (a) Scarab at high pose (small wheel base), (b) Scarab at low pose (large wheel base)

The concept of inching is not unique and has been investigated in the past. At the Army Land Locomotion Laboratory (Czako, 1963), the concept of a segmented vehicle with the ability to inch was introduced. It was determined through theoretical analysis that by keeping one axle stationary and propelling the other forward, the thrust generated by the stationary tires would be transferred to the rolling tires allowing them to better overcome the resistance on that axle. The stationary tires would not encounter as much resistance as they would when rolling, thus the net resistance on the vehicle as a whole decreased while the net thrust remained the same. In theory this would certainly allow an inching vehicle to generate more net work than a pure rolling vehicle; however further experimental work is required. The research performed in this paper aims to build upon these principles and gain a better understanding of how push-pull locomotion (specifically inching) can affect vehicle performance in soft terrain.

\section{EXPERIMENTAL COMPARISON BETWEEN ROLLING AND INCHING}

A series of experiments were conducted in the SLOPE laboratory at NASA GRC to quantitatively compare the performance of rolling to inching, a subset of push-pull locomotion. One major challenge for off-road vehicles, especially on the Moon or Mars, is having to traverse steep slopes in soft soil. The drawbar pull test can be used to predict a vehicle's slope-climbing performance (Freitag, 1970). This test consists of driving a vehicle across a prepared terrain while applying resistance opposite the direction of travel (the drawbar pull force) and observing how the 
vehicle's driving performance is affected. Drawbar pull force is essentially the external work that a vehicle can generate, which is indirectly related to its ability to climb a slope.

\section{Test Description}

Two different sets of tires were used with the Scarab rover for these comparative tests: 28 " diameter X 7" wide rigid tires and 28" diameter X 7" wide rubber pneumatic tires (Figure 3). The tread on the pneumatic tires was shaved down to create a low-profile flat cross section, while sandpaper-like tape was added to the rigid tires to allow for better gripping between the tire and soil. These two types of tires represent the two extremes of compliance and footprint size. The rigid tires do not conform to the terrain and sink more in soft soil. The pneumatic tires at 2 psi are very compliant and only disturb the soil minimally. From past experiments, it is evident that compliant tires provide much better drawbar pull on soft soil than rigid tires, as the larger footprint is associated with greater thrust and lower resistance (Wong, 2011). Because both the rolling and static tires participate when inching, it was important to test both rigid and compliant tires for this case.

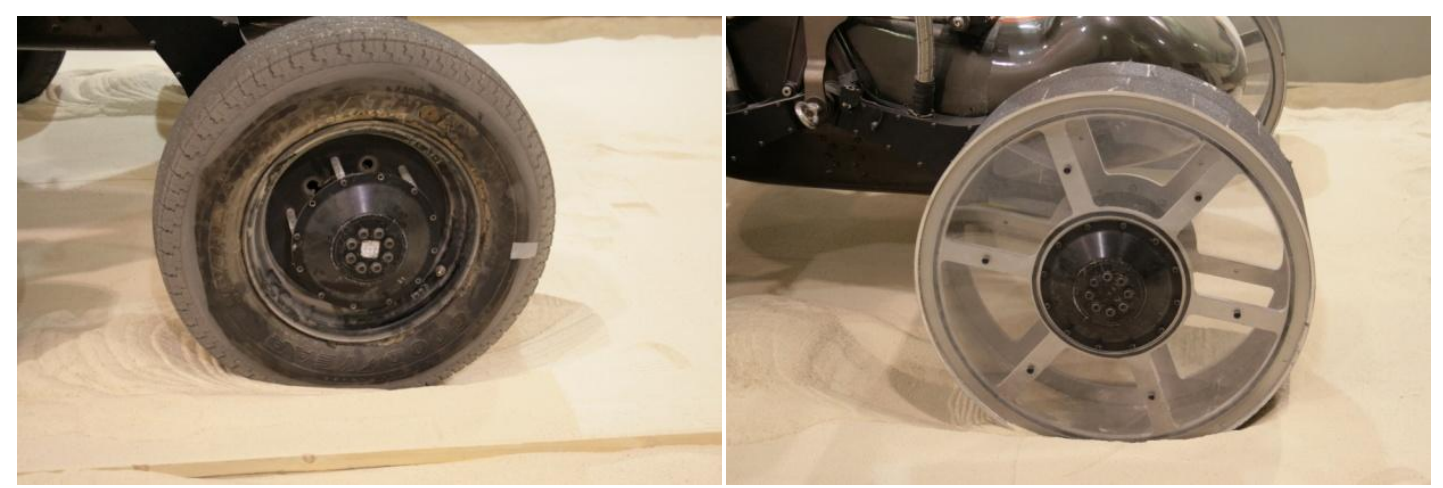

Figure 3: (a) Rubber 28" X 7" pneumatic tire without tread (2psi), (b) Rigid 28" X 7" tire without tread

To simulate difficult driving conditions on the Moon, the drawbar pull tests were conducted in a soil bin approximately $8 \mathrm{~m} \times 3 \mathrm{~m} \times 0.3 \mathrm{~m}$ filled with GRC-1, a Lunar terrain strength simulant, primarily composed of silica sand (Oravec, 2010). This simulant was prepared before each test to a highly-repeatable standard condition by loosening and leveling the terrain. A cable was attached to a hitch at the rear wheel hubs of Scarab through a load cell (see Figure 4). The cable was wrapped around a drum which was coupled to a motor through a magnetic controlled clutch, thus the torque on the drum could be controlled by varying current to the clutch. Using feedback from the load cell, the tension in the cable was computer controlled to achieve constant drawbar pull forces on the vehicle. Scarab would begin driving at one end of the soil bin while the load would be increased in steps over consistent intervals. For the case of rolling, the load would increase approximately every $0.5 \mathrm{~m}$; for the case of inching, the load would be held constant for at least two inching cycles, and then increased. For each type of tire and driving mode, a full set of drawbar pull forces would be applied, ranging from zero to approximately $40 \%$ of the vehicle's weight $(\sim 1600 \mathrm{~N})$. 


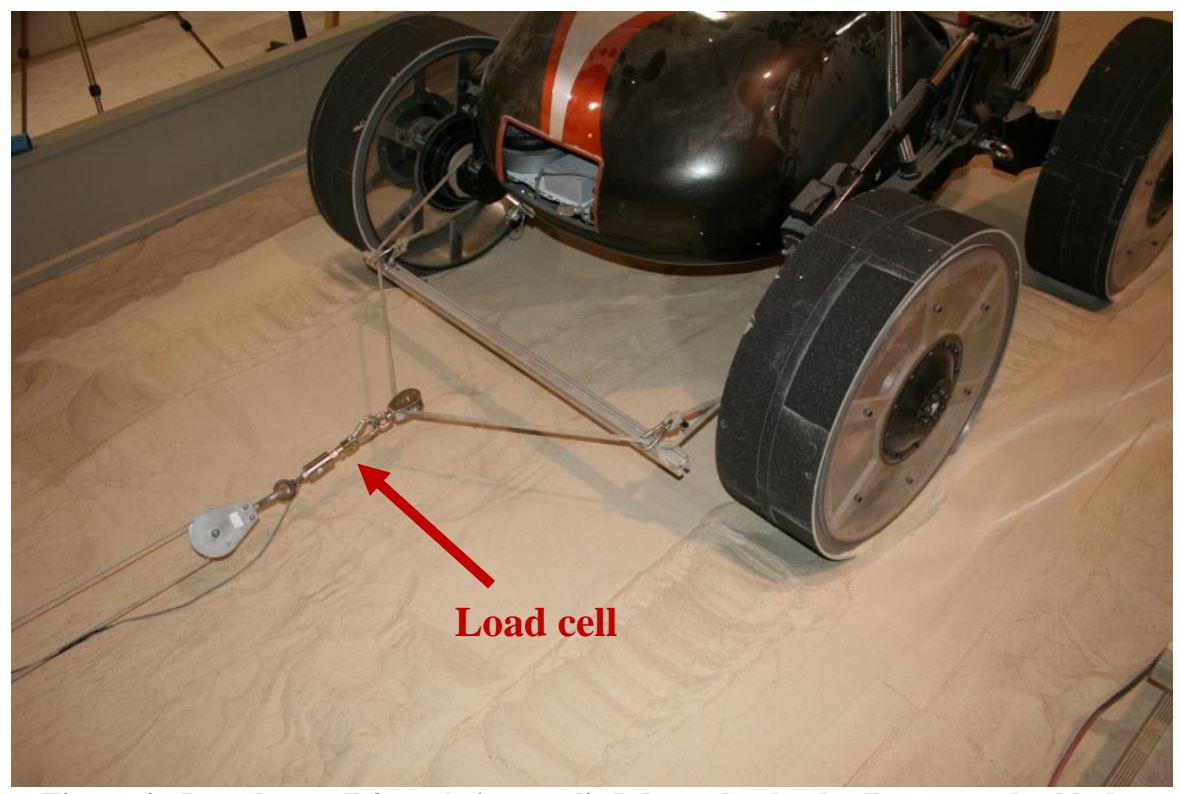

Figure 4: Drawbar pull force being applied through a load cell at rear wheel hubs

\section{Results}

The metric travel reduction was chosen for evaluating these two modes. It can be defined as the reduction in vehicle velocity for a given drawbar pull force relative to the vehicle's self-propelled velocity (no drawbar pull force), normalized to the selfpropelled velocity (assuming constant wheel rotational velocity).

$$
T R=\frac{V_{s p}-V_{\text {actual }}}{V_{s p}} \times 100 \%
$$

$$
\begin{aligned}
& T R=\text { Travel Reduction }(\%) \\
& V_{s p}=\text { Self-propelled velocity of vehicle }(\mathrm{m} / \mathrm{s} \text { or } \mathrm{m} / \text { cycle }) \\
& V_{\text {actual }}=\text { Velocity of vehicle for given drawbar pull force }(\mathrm{m} / \mathrm{s} \text { or } \mathrm{m} / \text { cycle })
\end{aligned}
$$

Travel reduction describes the vehicle's performance as a whole in terms of efficiency and safety; higher TR corresponds to higher soil displacement, thus more wheel sinkage, increasing the likelihood of the vehicle to become immobilized. For the case of rolling, velocity was measured using a robotic tracking system and averaged over a given length. For inching, because the vehicle velocity is not constant throughout each cycle, the velocities were calculated as distance per cycle. In both cases, the velocity components of the TR equation cancel out, leaving a unitless value for travel reduction.

For each region in which the drawbar pull force (DP) was held constant, both TR and DP (normalized to vehicle weight) were averaged over a known distance or for the case of inching, multiple cycles. The results are shown in Figure 5. For the pneumatic tires, there is much lower TR (better performance) when rolling compared to inching at DP forces less than 0.25. However, at greater DP, inching mode 
provides lower TR and a much higher maximum DP is achieved. The maximum DP that the pneumatic tires can generate while inching is approximately 0.37 compared to 0.28 when rolling, a $32 \%$ increase in pull force.

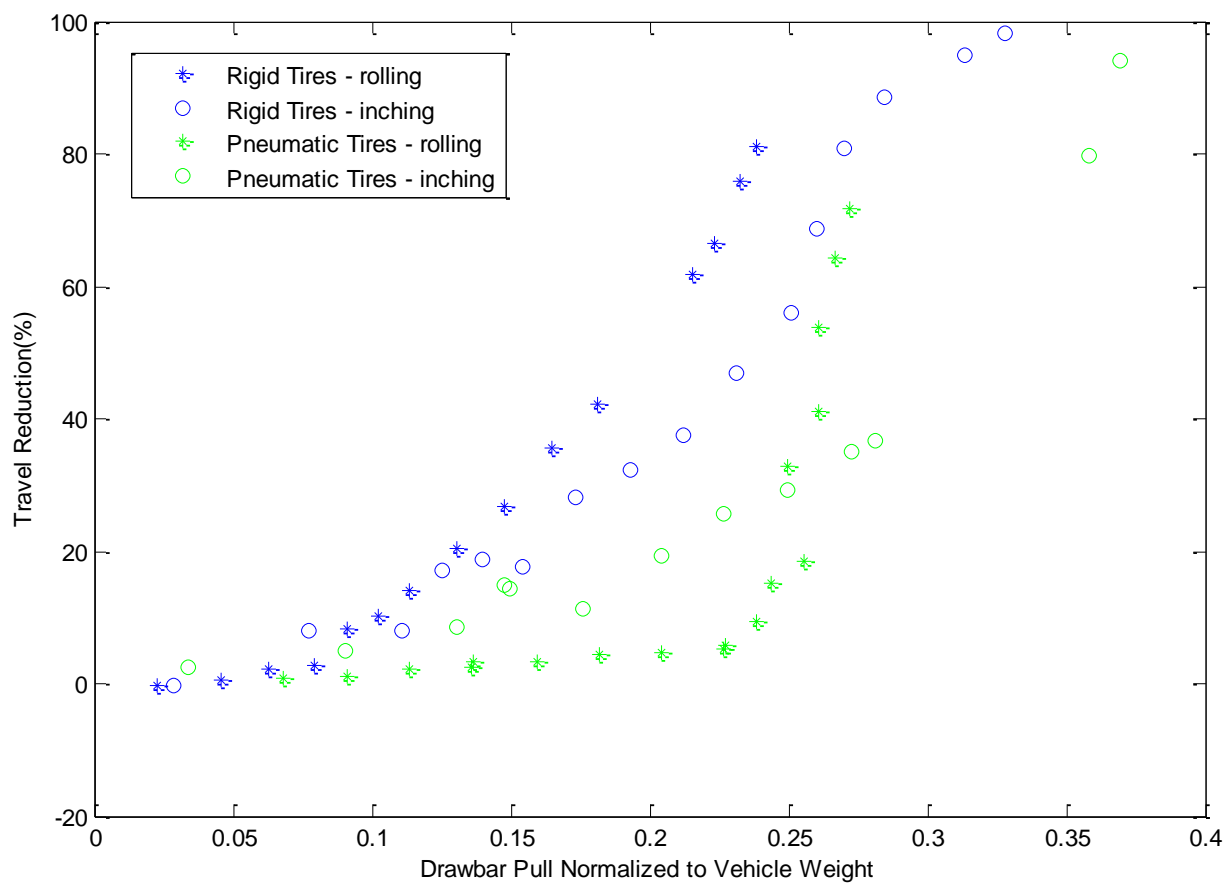

Figure 5: Comparison of rolling vs. inching for compliant and rigid tires in loose GRC-1

For the rigid tires, on the other hand, there isn't a region where rolling mode shows a clear advantage over inching mode. The TR of rolling and inching is similar at low DP, and inching produces much lower TR after DP is increased past 0.15 . The maximum inching DP force is about $30 \%$ higher than rolling. When using inching mode, the compliant tires still perform better than the rigid tires, though this difference is not as severe compared to rolling mode. The shapes of the inching curves are different than typical rolling drawbar pull curves as well, indicating that a different mode of traction is occurring between the vehicle and terrain.

\section{UNDERSTANDING SOIL RESPONSE USING VISUAL ANALYSIS}

A system for understanding the interaction between terrain and tires has recently been developed called the Shear Interface Imaging Analysis Tool (Moreland, 2011). This system consists of a small soil bin with a clear glass wall against which a tire can be positioned (see Figure 6). The soil bin in this case has been filled with GRC-1 and prepared to a repeatable condition by loosening, leveling, and compacting the soil. A rigid wheel with a diameter 1/3 the full scale wheels on Scarab was placed up against the inside of the glass so that the interface between the wheel and terrain could be seen easily. In order to simulate soil directly underneath the center of the tire, a width of 1/6 the full size wheel was used; in other words the glass wall was virtually placed through the center of the tire in the width direction. By assuming the 
soil response is symmetric about the center of the tire in the width direction, the glass wall was determined to not have a significant effect on the soil response as long as friction forces between the glass and soil were minimal (Wong, 1967).

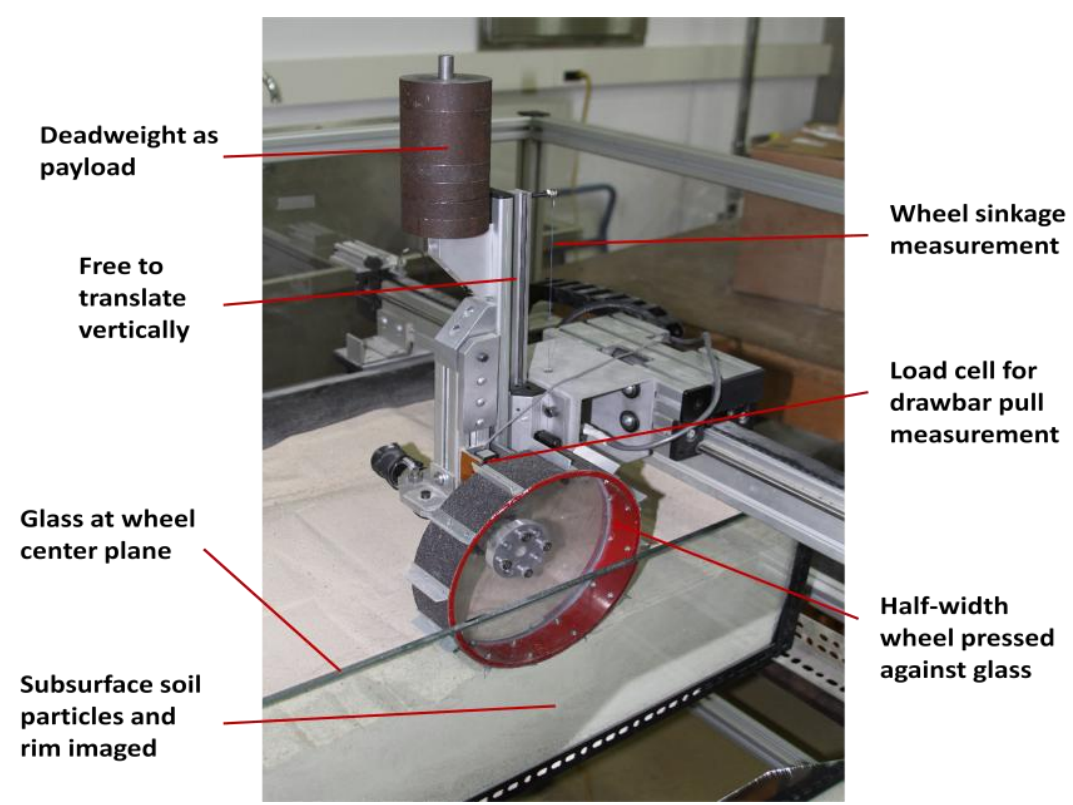

Figure 6: Soil Imaging Rig with rigid tire in clear soil tank with GRC-1

The wheel is attached to a motor which can be locked, driven, or free-rolling. The motor is mounted to a vertical shaft riding on bearings which allows the wheel to sink freely. This vertical shaft rides along a carriage that is actively controlled in the horizontal direction to simulate the vehicle travel. By controlling both the rotational speed of the wheel and the translational speed of the carriage, wheel slip can be induced. For these specific tests two types of travel were simulated. For the first case, the wheel and carriage were driven at speeds to create a noticeable amount of shearing in the terrain below the tire, essentially simulating a conventional rolling wheel under DP. For the second case, the wheel rotational speed was held at zero (braked) while the carriage was moved horizontally a short distance, towing or pushing the wheel. This produced a situation similar to inching where the stationary wheels generate thrust by pushing off of the soil in a horizontal direction.

A camera was mounted outside of the glass wall and coupled with the carriage so that it would move laterally with the wheel. High-resolution photos were taken at a rate of approximately eight frames per second during the tests to image the soil motion beneath the tire. The images could be read into a program where, by using optical flow and clustering techniques, individual soil particles were tracked in two dimensions (Moreland, 2011). The velocities of these particles were determined throughout a test and shearing patterns were observed.

Examples of the soil shearing patterns for the two cases can be seen in Figure 7 (particle velocity magnitude) and Figure 8 (particle velocity direction). In both cases the simulated motion of the vehicle is to the right; the rolling wheel is driving clockwise and the sliding wheel moves to the left slightly to push off of the terrain. Large differences in the direction and shape of the shearing patterns are evident. The 
soil beneath the rolling wheel appears to follow the shape of the wheel in a direction tangential to the wheel rotation. The failure occurs close to the tire-terrain contact and is fairly uniform. This type of soil response was defined by Bekker as "grip" failure" (Bekker, 1960). The braked wheel, however, produces a much different response in the soil. The soil displacement occurs as a bulk quantity moving at once in a single direction. Bekker describes this soil response as "ground failure" or "general shear failure" (Bekker, 1960).

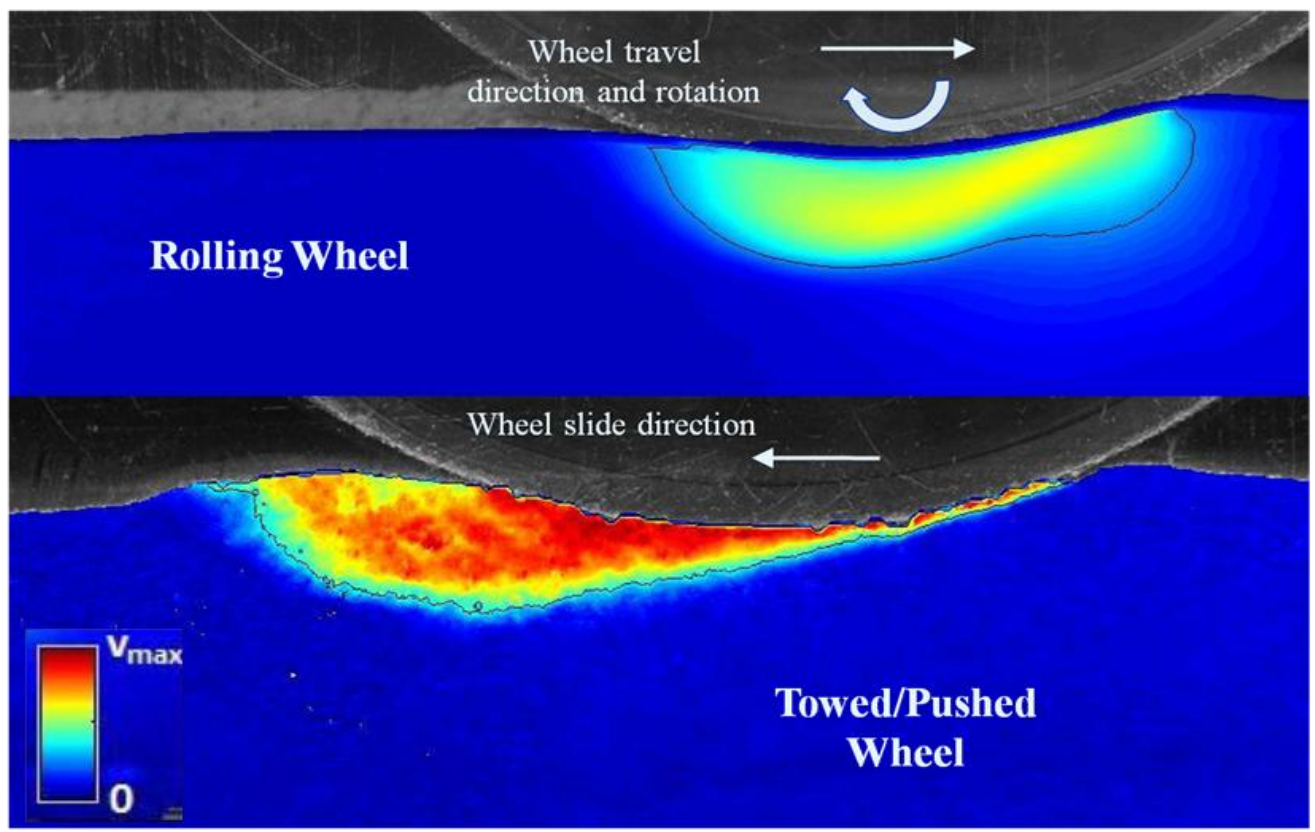

Figure 7: Soil velocity response to Rolling Wheel vs. Pushed Wheel (Moreland, Skonieczny, Wettergreen, Asnani, \& Creager, 2011); color indicates magnitude of soil particle velocity

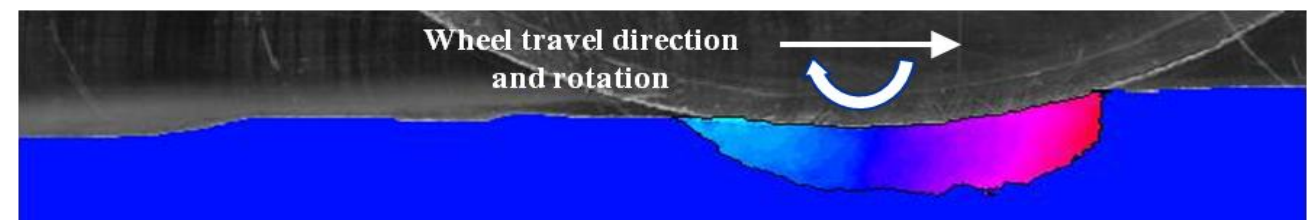

Rolling Wheel

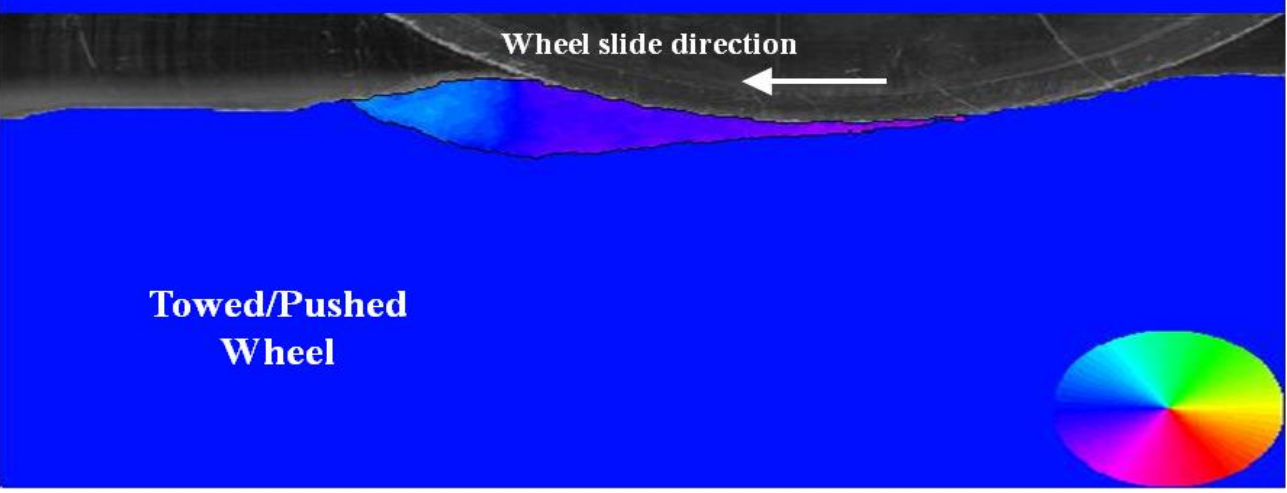

Figure 8: Soil directional response to Rolling Wheel vs. Pushed Wheel; color indicates direction of soil particle velocity 
For these specific examples, the magnitude of the particle velocities cannot be used for comparison because different amounts of thrust are being generated here. However, the direction of the soil particles being displaced provides valuable insight. For rolling, only a certain portion of the particle motion is in the direction of thrust. Much energy is spent moving the soil vertically. The constant changing of particle direction requires more wheel motion to compact the soil and generate thrust. The braked wheel, on the other hand, creates soil displacement almost exclusively in the direction of thrust. Soil compaction is produced more quickly because the soil particles can only move in one direction. Pushing or pulling a braked wheel appears to be a much more efficient method of generating large amounts of thrust. This difference in soil failure could help explain the observed differences in performance for rolling vs. inching. Adding to previous theories which claimed inching and other methods of push-pull locomotion exhibited more DP capability over rolling by decreasing the wheel resistance (Czako, 1963), the image analysis shown here indicates that an entirely different mode of soil response occurs when inching, producing an increase in thrust force as well.

\section{CONCLUSIONS/SUMMARY}

From early drawbar pull tests and Shear Interface Imaging Analysis, it is evident that push-pull locomotion can be very useful when traversing frictional terrain where high net force must be generated. Significant decreases in travel reduction at high DP when inching correspond to a lower risk of becoming immobilized on steep slopes or in soft soil. Higher drawbar pull forces can be achieved while inching, which corresponds to increased mobility and towing capacity.

It is also evident that inching is not the most efficient or useful mode of locomotion at low loads, especially for compliant tires. It is suggested that inching (and likely other forms of push-pull locomotion) be used as a fail-safe option during missions. A vehicle could push its limits of conventional rolling when attempting to traverse a difficult environment; then if it reaches the point of becoming immobilized, it could switch to inching or another form of push-pull locomotion to get itself free or continue on its path. The ability to use push-pull locomotion would be incredibly beneficial, especially for autonomous robots traversing unknown terrain such as on the Moon or Mars.

It is recommended that the next step be to develop vehicle controls for inching mode, focusing on coordinating wheel base translational and wheel rotational rates. In additional, other metrics should be considered for comparing rolling and push-pull locomotion. Finally, inching locomotion should be systematically tested for overcoming rolling mobility limitations, such as in hill climbing and for extrication from soft terrain.

\section{REFERENCES}

Bekker, M. G. (1960). Off-the-road Locomotion. Ann Arbor, MI: The University of Michigan Press. 
Czako, T. F., Janosi, Z. J., \& Liston, R. A. (1963). An Analysis of Multi-element Inching Vehicles. Center Line, MI: U.S. Army Tank-automotive Center, Land Locomotion Laboratory.

Freitag, D. R., Green, A. J., \& Melzer, K. J. (1970). Performance Evaluation of Wheels for Lunar Vehicles. Vicksburg, Mississippi: U.S. Army Engineer Waterways Experiment Station.

Moreland, S., Skonieczny, K., Wettergreen, D., Asnani, V., \& Creager, C. (2011). Soil Motion Analysis System for Examining Wheel-Soil Shearing. 17th International Conference for the Society of Terrain-Vehicle Systems. Blacksburgh, VA.

NASA Jet Propulsion Laboratory. (n.d.). JPL Robotics. Retrieved from The ATHLETE Rover: http://www-robotics.jpl.nasa.gov/systems/system.cfm?System=11 Oravec, H., Asnani, V., \& Zeng, X. (2010). Design and characterization of GRC-1: A soil for lunar terramechanics testing in Earth-ambient conditions. Journal of Terramechanics, 47(6) , 361-377.

Wettergreen, D., Moreland, S., Skonieczny, K., Jonak, D., Kohanbash, D., \& Teza, J. (2010). Design and field experimentation of a prototype Lunar prospector. International Journal of Robotics Research, Vol. 29 no. 12 pg. 1550-1564.

Wong, J. Y. \& Reece, A. R. (1967). Behavior of Soil Beneath Rigid Wheels. Journal of Agricultural Engineering Research, Vol. 12, No. 4, pp. 257-269. Sons, Inc.

Wong, J. Y. (2001). Theory of Ground Vehicles. New York, NY: John Wiley \& 